\title{
The use of radiation-resistant materials in gas semiconductor Fourier spectroscopy for express analysis of rocket fuel in the production process on the Moon / Mars / asteroids
}

https://doi.org/10.1515/astro-2021-0014

Received Oct 25, 2021; accepted Nov 20, 2021

\begin{abstract}
The paper considers the possibility of using thin films based on samarium sulfide, as well as composite systems of metal-oxide semiconductors doped with rare-earth elements, as radiation-resistant sensitive materials for monitoring leaks of rocket fuel components. The data of electrophysical studies of the obtained sensor layers were processed within the framework of the semiconductor Fourier spectroscopy constructed in relation to gas-analytical systems and the nonlinear regression algorithm of selective calibration. The latter solves really the problem of sensors' selectivity including distinguishing the substances of the same homology such as methane, propane and n-hexane.
\end{abstract}

Keywords: radiation resistance, gas sensor, rare earth semiconductors, Fourier transform, nonlinear regression

\section{Introduction}

Semiconductor resistive gas sensors are one of the main types of chemical sensors used in industry, including in the rocket and space industry. Their principle of operation is based on the change in the conductivity of the sensitive layer when exposed to the components of the gas medium. The advantages of such sensors are: high sensitivity, high speed, as well as low cost in their mass production. At the same time the study of the mechanisms of particle adsorption on the surface of a gas sensor has not yet led to the solution of the fundamental triune problem of increasing the selectivity, sensitivity, and stability of sensors. Currently, various methods are being actively developed that could solve these problems, much attention is paid to the application of the chemometric approach to the analysis of adsorption data (Gwiźdź et al. 2015; Alizadeh 2010; He et al. 2017; Shahid et al. 2018). Recently (Kazakov et al. 2020), a promising approach in the field of semiconductor gas analysis was proposed, based on a modification of optical methods of

Corresponding Author: Sergey Kazakov: Laboratory of Physics of Rare-Earth Semiconductors, Ioffe Institute, 26 Politekhnicheskaya, St Petersburg, 194021, Russian Federation;

Email: kazakov59@mail.ioffe.ru

Mikhail Grevtsev, Victor Chistyakov, Sergey Solov'ev: Laboratory of Physics of Rare-Earth Semiconductors, Ioffe Institute, 26 Politekhnicheskaya, St Petersburg, 194021, Russian Federation

ə Open Access. @ 2021 S. Kazakov et al., published by De Gruyter. (cc) BY License
Fourier spectroscopy, which makes it possible to unambiguously solve the problem of increasing the selectivity of a gas sensor. The essence of the approach is to obtain the spectral characteristic of the sensor from the total change in its electrical conductivity over time with an increase (decrease) in the sensor surface temperature in the analyzed medium by applying a discrete Fourier transform to the obtained data. The use of an appropriate algorithm for processing experimental data makes it possible to isolate from the useful detector signal the amplitude-frequency spectrum of the concentration effect on the semiconductor conductivity of a particular component of the gas mixture. The paper (Chistyakov et al. 2021) describes a method for the selective determination of trace concentrations of impurities in air, based on statistical modeling of the signal of changes in electrical conductivity under temperature modulation of a chemical sensor. Identification and determination of the gas concentration is carried out after drawing up its selectivity portrait, which has a characteristic appearance for each substance.

The development of semiconductor sensors for detecting fuel leaks during tests of cryogenic engines is still an urgent task (Gutman et al. 1993; Pijolata et al. 2002). One of the main requirements for materials used in the space industry, not only structural, but also functional, in particular, materials for determining the concentration of gases, is radiation resistance. Radiation resistance is usually understood as the invariance of the parameters of a semicon- 
ductor when exposed to nuclear radiation. Irradiation of semiconductors leads to the formation of deep centers in them - radiation defects of the acceptor or donor nature. The solution to the problem of radiation resistance of semiconductor materials can be doping them with rare earth elements (REE), which are characterized by a state with a variable valence. A change in the valence of the embedded REE leads to relaxation of radiation excitations (Kaminsky et al. 1995; Brehovskih et al. 1982). In this regard, some rare-earth semiconductors should also have high radiation resistance.

\section{Material and methods}

Semiconductor samarium sulfide (SmS) and tin dioxide-based composite materials (for example, the $\mathrm{SnO}_{2} / \mathrm{V}_{2} \mathrm{O}_{5} / \mathrm{La}_{2} \mathrm{O}_{3} / \mathrm{Pt}$ system) were used as the working layers of the sensors. Thin films of samarium sulfide were obtained by the sol-gel method. The initial powder of samarium sulfide, the radiograph of which is shown in Figure 1, previously ground in an agate mortar to a fine state. Then the specified sample of SmS powder was mixed with a $20 \%$ solution of the polymer polypropylene carbonate in propylenglycoldiacetate to obtain a homogeneous paste of black and purple color. Annealing the suspension in a vacuum at a temperature of $250^{\circ} \mathrm{C}$ to $500^{\circ} \mathrm{C}$ leads to the decomposition of the polymer to $\mathrm{CO}_{2}$ and $\mathrm{H}_{2} \mathrm{O}$. The solvent then evaporates and a layer of $\mathrm{SmS}$ is deposited on the surface SmS (Kazakov et al. 2015).

Semiconductor materials based on tin dioxide $\left(\mathrm{SnO}_{2}\right)$ were obtained by hydrothermal synthesis. The material was deposited from a solution of $\mathrm{SnCl}_{4} \cdot 5 \mathrm{H}_{2} \mathrm{O}, \mathrm{Na}_{3} \mathrm{VO}_{4} \cdot 12 \mathrm{H}_{2} \mathrm{O}$ and $4 \% \mathrm{LaCl}_{3} \cdot 7 \mathrm{H}_{2} \mathrm{O}$ with an ammonia solution to a $\mathrm{pH}$ value of 6.5-7.0 at room temperature, while continuous mixing was carried out using a magnetic stirrer. The resulting precipitate was separated by centrifugation and thoroughly washed from the chloride ions with distilled water until the indicator reaction to silver ions $\left(\mathrm{Ag}^{+}\right)$disappeared. The resulting suspension of the material was placed in an autoclave and kept for 6 hours at a temperature of $180^{\circ} \mathrm{C}$. After that, the suspension was dried, then sieved through a nickel sieve and calcined in a furnace at $600^{\circ} \mathrm{C}$ for 2 hours. The material was then platinated to obtain a material composition of $0.15 \%$ Pt.

The data of X-ray diffraction analysis of the obtained powder are shown in Figure 2. After the introduction of the modifier, the parameters of the unit cell of the crystal lattice of the semiconductor used as the main material change in comparison with the library data. This fact indicates the

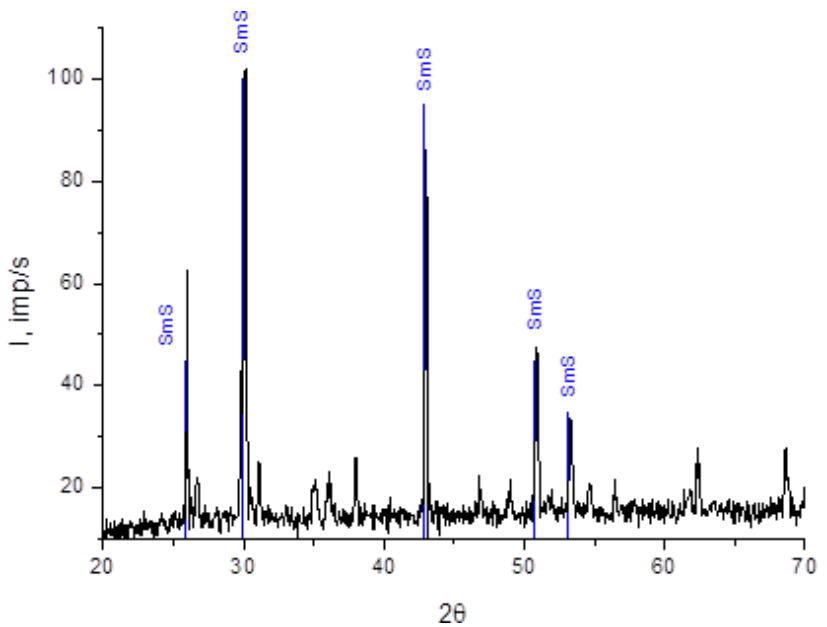

Figure 1. Diffractogram of polycrystalline SmS.

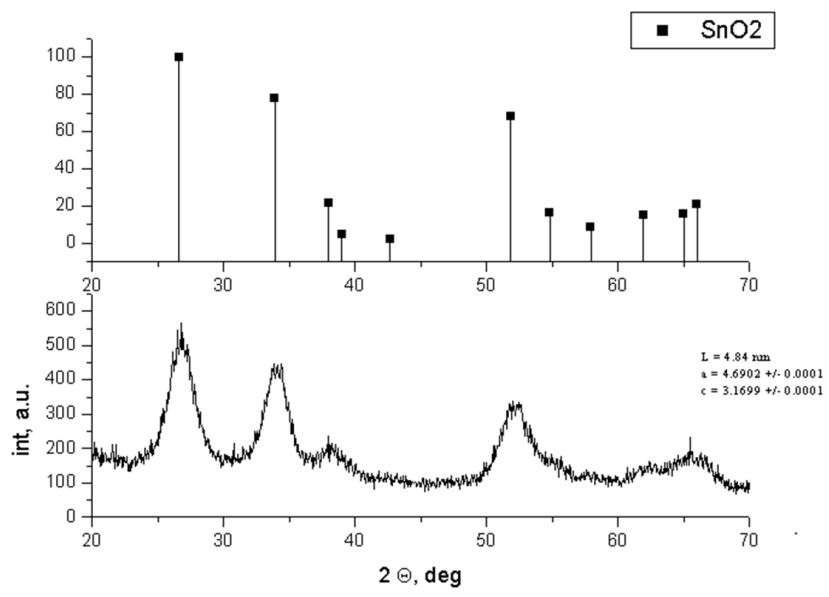

Figure 2. Diffractogram of the system $\mathrm{SnO}_{2} / \mathrm{V}_{2} \mathrm{O}_{5} / \mathrm{La}_{2} \mathrm{O}_{3} / \mathrm{Pt}$.

introduction of alloying additives into the structure of the crystal lattice to form a solid solution and the appearance of additional adsorption centers in the material, which are responsible for the conductivity of the sample and its gas sensitivity to a particular component of the gas mixture.

$\mathrm{X}$-ray phase analysis of the samples was carried out on a diffractometer DRON-4 ( $\theta-2 \theta$-scanning, $\mathrm{Cu}_{K \alpha}$-radiation, $\lambda$ $=1.54 \AA$ ).

\section{Theory and calculation}

\subsection{Calibration algorithm for semiconductor gas sensors using Fourier transform}

To study the electrophysical properties, the synthesized materials were applied to a microelectronic chip with a closed autonomous heater, which was a layer of compos- 
ite material consisting of platinated nanoscale aluminum oxide particles and borosilicate glass. On the surface of the chip there were platinum electrodes, between which there was a semiconductor gas-sensitive layer of the sensor. The experiment consisted in periodic scanning of the detector signal by temperature: the electrical conductivity of the sensor was measured under conditions of periodic heating and cooling of the gas-sensitive element at a given gas concentration. Then, by applying Fourier transforms to these data, an optical analog of the spectral characteristic of the gas sensor is obtained. The relative electrical conductivity of the detector $\left(\sigma(t) / \sigma_{0}\right)$ under this consideration is the optical analog of the relative intensity of the absorbed radiation $\left(I(t) / I_{0}\right)$, and the frequency response of the sensor expressed in units of $k T(t)$ is the optical analog of the frequency response of the energy of the absorbed radiation $h v$. The Fourier-series expansion of the sensor output signal, followed by the consideration of the lower harmonics of the resulting function, allows you to select the components and determine their concentrations. The frequency response of the sensor is responsible for the selectivity and stability of its operation (this is evidenced by the absence of a frequency shift in the sensor characteristics both during calibration and measurement). At the same time, the amplitude of the selected frequency is proportional to the concentration effect of a particular measured component of the gas mixture.

This section presents experimental data on the calibration of sensors for gasoline, solvent vapor, toluene, and methane, obtained by recording measurements of the electrical conductivity of the gas-sensitive sensor layer over time, both during heating and cooling. When preparing gas mixtures, certified brands of substances were used, and the preparation of calibration gas mixtures was carried out on certified equipment.

Figure 3 shows the difference signal of the change in the conductivity of the sensor over time during cooling for three given constant concentrations of gasoline.

The tables in the upper corner of Figure 3 show the trend lines and the parameters of the trend line equation. Below are the equations of the trend lines in the areas with peaks:

$$
\begin{aligned}
& G 51(t)=-3.87033 \cdot 10^{-4} \cdot t^{2}+2.41392 \cdot t-3750.53567 \\
& G 31(t)=-1.67449 \cdot 10^{-4} \cdot t^{2}+1.0479 \cdot t-1631.22129 \\
& G 21(t)=-1.29796 \cdot 10^{-4} \cdot t^{2}+0.81399 \cdot t-1270.73711
\end{aligned}
$$

The Fourier-images of the trend lines for three different gasoline concentrations are presented below in graphical form (see Figure 4(a) and Figure 4(b)). The time extremum point for all the presented functions turned out to be the same, $\sim 3125 \mathrm{~ms}$, which is further confirmed by the graph for the first derivatives of these functions (see Figure 5).

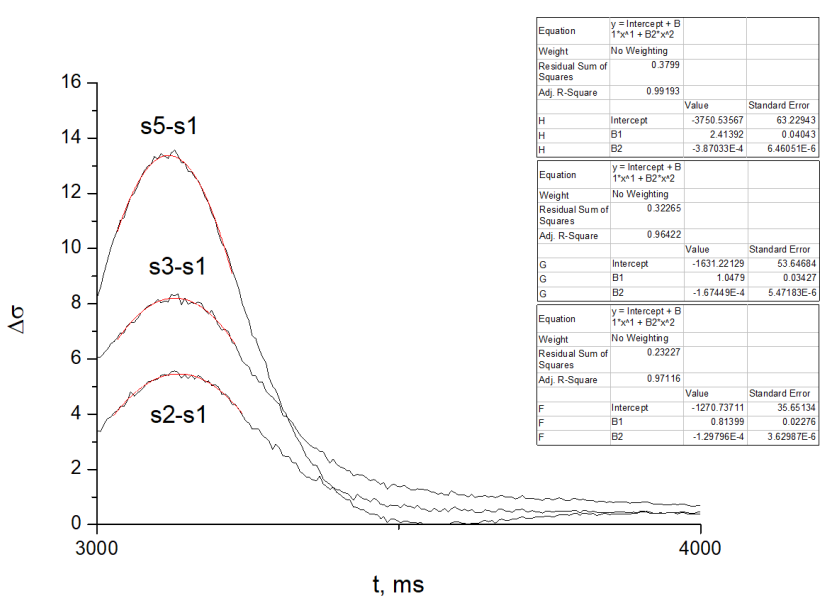

Figure 3. Change in conductivity over time for a semiconductor SmSbased sensor at specified gasoline concentrations.

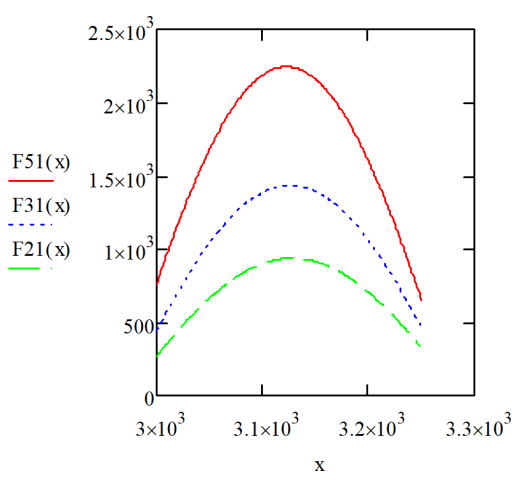

(a)

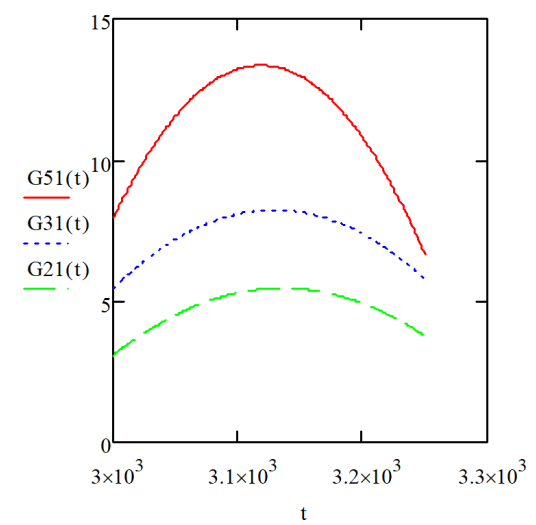

(b)

Figure 4. A graphical view of the Fourier images (graph (a)), and the trend line functions (graph (b)) for three different gasoline concentrations. 


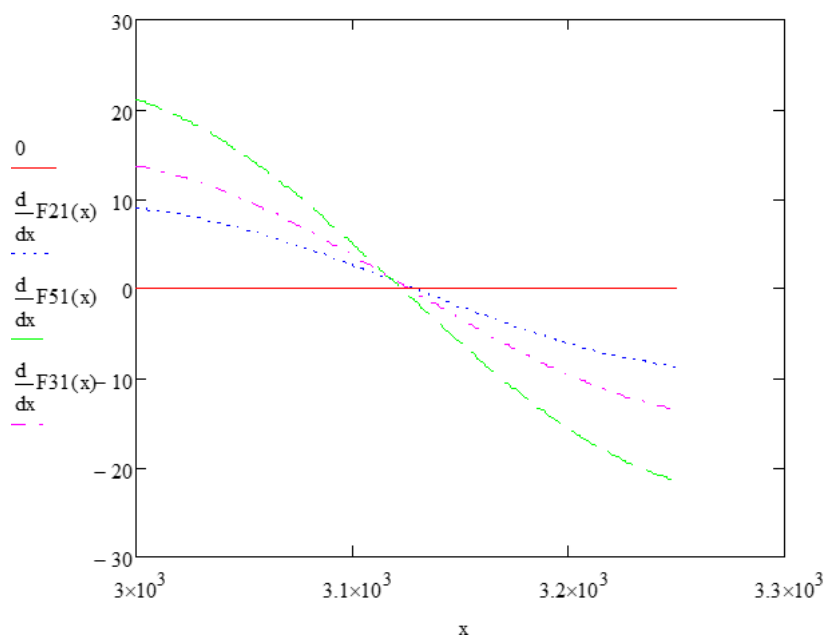

Figure 5. The first time derivatives of the Fourier-images for three different concentrations of gasoline.



Figure 6. The square of the amplitude of the real part of the spectral characteristic of the sensor, proportional to the concentration of the measured component in the gas mixture.

Plotting the square of the amplitude of the real part of the spectral characteristics of the Fourier transforms as a function of frequency, we see that for all concentrations the frequency is the same, $\sim 0.036 \mathrm{~s}^{-1}$ (see Figure 6).

According to the above calculation algorithm (for the case of sensor cooling), we calculated the frequency response of the sensor when it is heated. The value of the process frequency equal to $\sim 0.040 \mathrm{~s}^{-1}$, is obtained, which is quite close to the value of $\sim 0.036 \mathrm{~s}^{-1}$ within the experimental error. Thus, the optimal temperature for detecting gasoline vapors can be determined both during the heating process and during the cooling process of the sensor.

The calibration algorithm described above was also used in the processing of experimental data obtained on the same sensor after calibration for other gases. Figure 7 shows the difference signal of the change in the conductivity of

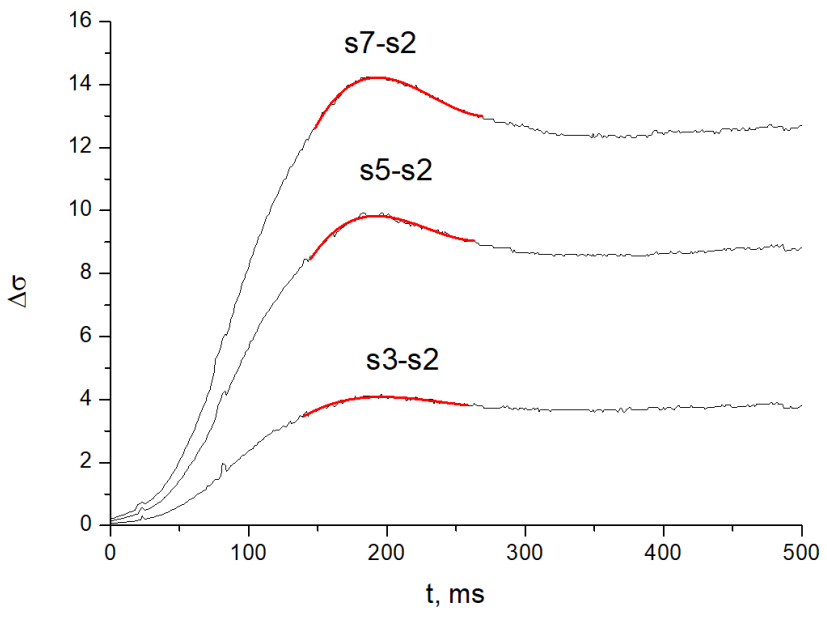

Figure 7. The difference signal of the change in the conductivity of the SmS-based-sensor over time during its heating at three different concentrations of solvent vapors in atmospheric air.

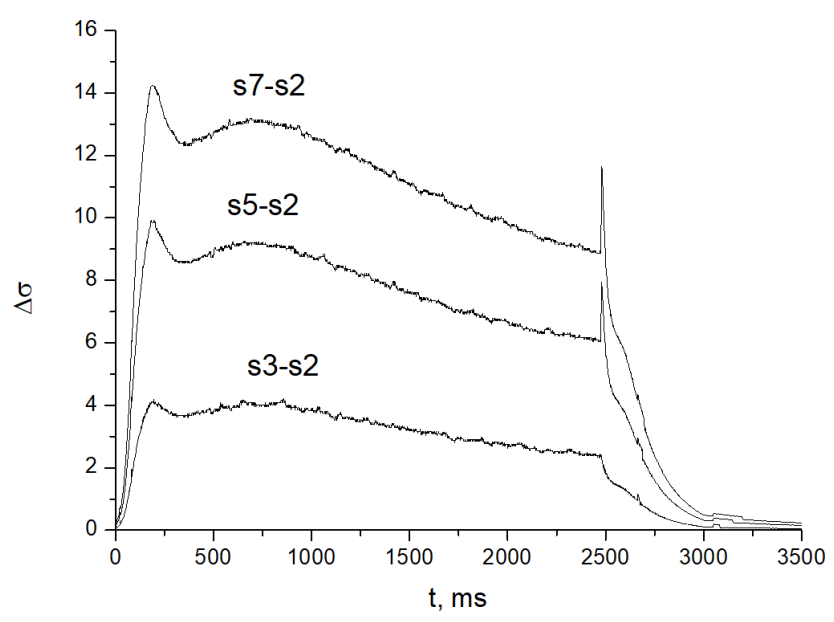

Figure 8. Time difference signal of the SmS-based sensor conductivity change for three different concentrations of solvent vapors in atmospheric air (heating and cooling).

the sensor over time during its heating at three different concentrations of solvent vapors.

The extremum point in Figure 8 corresponds to the 170th millisecond of the process. At the same time, the frequency estimate gives a value of $\omega \sim 0.459 \mathrm{~s}^{-1}$, which is about an order of magnitude higher than for gasoline vapors. Below (see Figures 8-10) provides sensor calibration data for various volatile hydrocarbons contained in atmospheric air.

The values of the frequency characteristics of the sensor correspond in order of magnitude to the values of the rate constants of desorption of molecular gases from the surfaces of oxide semiconductor sensors: $\mathrm{k}_{2} \sim 0.05 \mathrm{~s}^{-1}$ (Kazakov et al. 2015), which is confirmed by the study of composite materials (in particular, tin dioxide doped with 


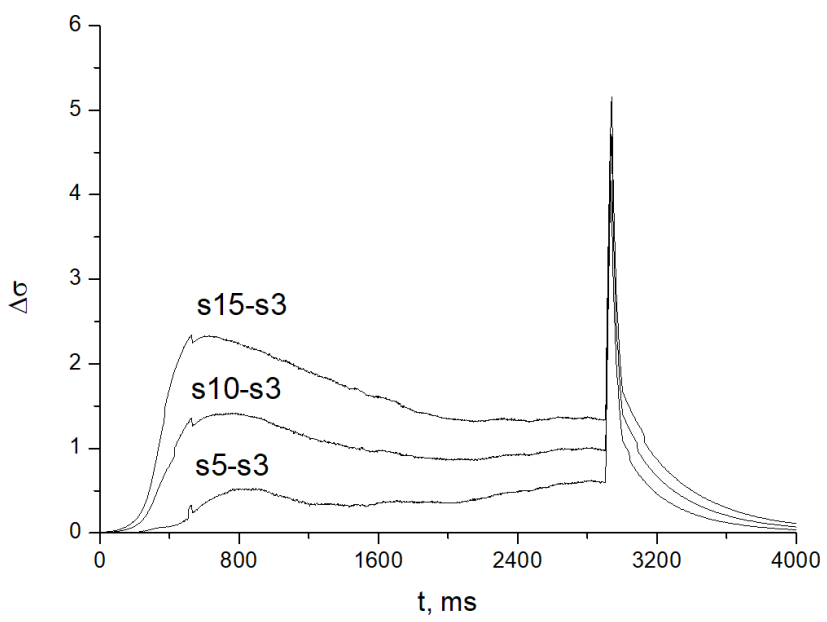

Figure 9. Time difference signal of the SmS-based sensor conductivity change for three different concentrations of methane vapor in atmospheric air (heating and cooling).

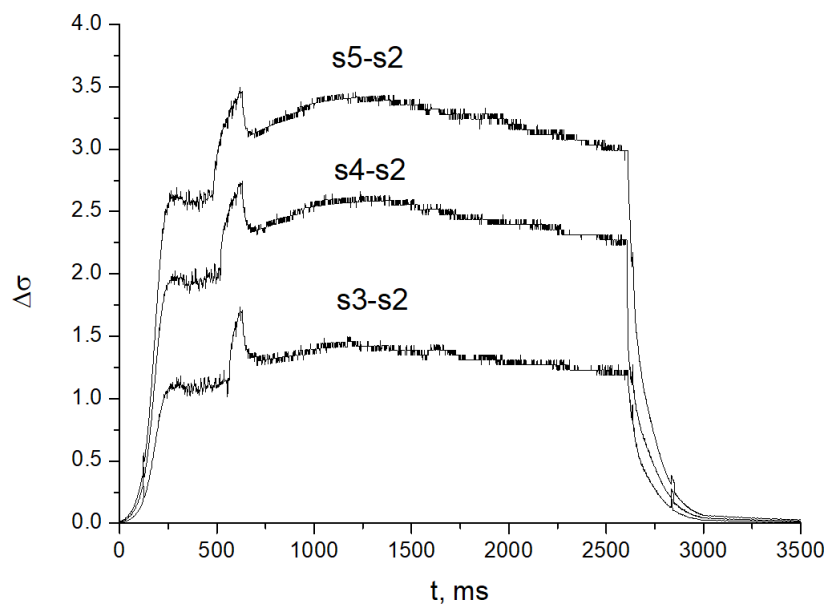

Figure 10. Time difference signal of the SmS-based sensor conductivity change for three different concentrations of toluene vapor in atmospheric air (heating and cooling).

lanthanum atoms). It can be assumed that the frequency characteristics of the sensors based on samarium sulfide obtained by us also correspond to the parameters of the rate constants of reverse chemisorption reactions for the hydrocarbons used in the experiments. The stability of the spectral characteristic of the sensor is confirmed by the absence of a shift in the characteristic frequency responsible for adsorption.

\subsection{Nonlinear regression algorithm for selective sensor calibration}

The response of the electrical conductivity of a semiconductor sensor in a medium with an active substance at a

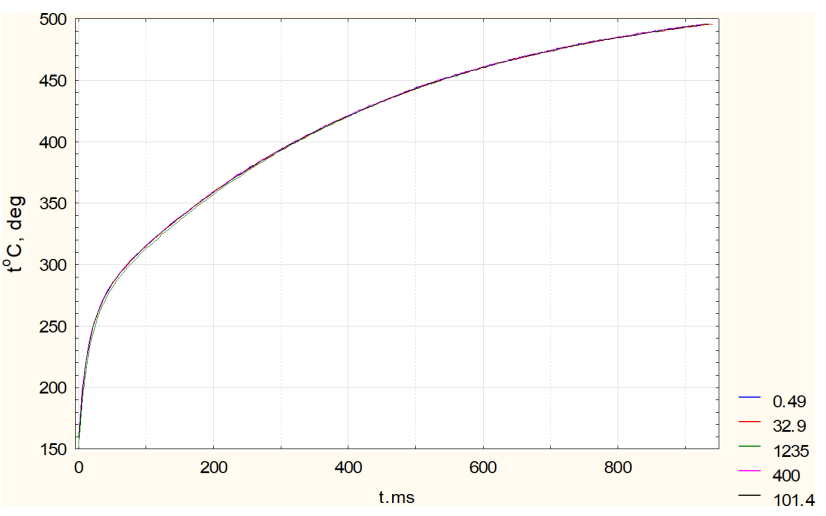

Figure 11. Temperature modulation graphs $\mathrm{T}(\mathrm{t})$ for different measurements.

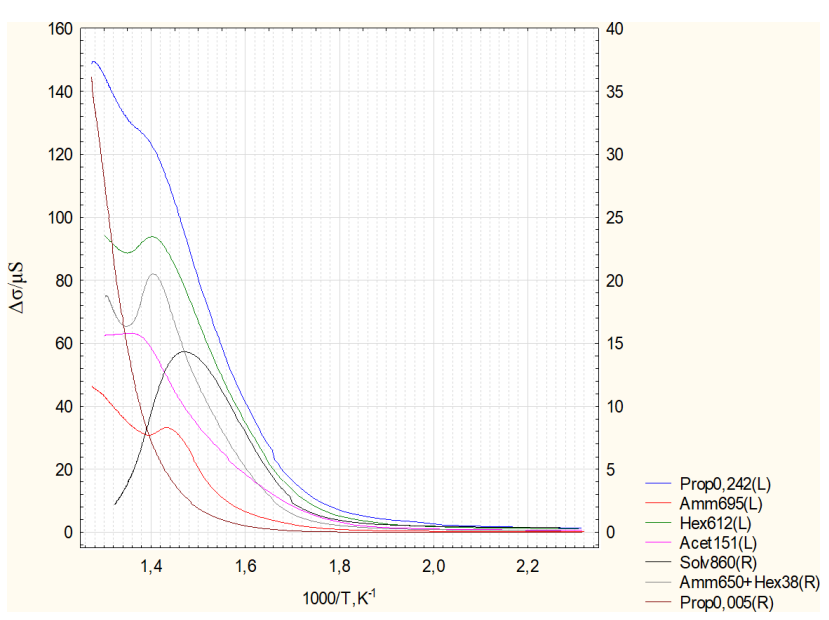

Figure 12. Different-shaped electrical conductivity signals as functions of the inverse absolute temperature $\Delta \sigma(z=1000 / T(t))$ for different adsorbents on $\mathrm{ZnO}$-based sensor.

rapid temperature change in a certain range from $T_{\min }$ to $T_{\max }$ carries information about chemisorption processes occurring on the surface at different speeds. The final signal $\Delta \sigma(t)$ at a given adsorbate concentration in the gas phase is a complex function and depends both on the surface properties of the semiconductor and on the heating mode $T(t)$ of the sensor surface. However, for the case of a fast heating mode reproducible from measurement to measurement, $T(t)$ (see Figure 11) it can be assumed that the response $\Delta \sigma$ is entirely determined by the dynamic equilibrium between the chemisorbed particles and their concentration in the volume, which is specific for each substance. This assumption allows us to consider the detector signal in coordinates $\Delta \sigma(z=1000 / T(t))$ (see Figure 12).

The main idea of the nonlinear regression method of calibration and processing of the sensor signal is as follows. Each curve of the sensor response to a certain concentration of a given substance in the considered gas medium 
(artificial air) when heated for $\sim 1 \mathrm{~s}$ from $150^{\circ} \mathrm{C}$ to $500^{\circ} \mathrm{C}$ is interpolated by a number of model dependencies of a special type - rational functions of the variable $z=1000 / T$, whose constant numerators are the main parameters, and whose denominators are reduced polynomials of degree 3 or 4.

$$
\begin{gathered}
F_{1}(z)=\frac{A_{1}}{z^{4}+b_{1} z^{3}+c_{1} z^{2}}, \\
F_{2}(z)=z F_{1}(z), \\
F_{3}(z)=\frac{A_{3}}{z^{4}+b_{3} z^{3}+c_{3} z^{2}+d_{3} z}, \\
F_{4}(z)=\frac{0.0001 A_{4}}{z^{4}+b_{4} z^{3}+c_{4} z^{2}+d_{4} z+h_{4}}, \\
F_{5}(z)=\frac{A_{5}}{b_{5} z^{4}+c_{5} z^{3}+d_{5} z^{2}+h_{5} z+1}, \\
F_{6}(z)=\frac{A_{6}}{b_{6} z^{4}+c_{6} z^{3}+d_{6} z+1} .
\end{gathered}
$$

The positive principal parameters $A_{i}>0, i=1 . .6$ should change rapidly with the concentration of $C$, while the coefficients of the polynomials in the denominators change slowly.

All sets of parameters are evaluated using the Levenberg-Marquardt method. When all the parameters are significant at the level of $\mathrm{P}=95 \%$, the concentration dependences $A_{i},() i=1 . .6$, that make up the calibration portrait of a certain substance are constructed for the main parameters.

These portraits may contain "bald" areas where some (but not all!) the parameters are not defined meaningfully. In general, the calibration portraits are individual for each substance. The continuity and smoothness of their curves $A_{i},() i=1 . .6$ are provided by sufficiently high values of the Student's criterion, $t\left(A_{i}\right)=10 . .60$, and the relative constancy of the coefficients in the denominators by even higher numbers from 100 to 20000 (!).

When detecting an unknown gas $\mathrm{X}$, a hypothesis is put forward $\mathrm{H}_{0}: \mathrm{X}=\mathrm{Y}$. In accordance with the hypothesis, the temperature range of signal processing is determined and, where possible, the main parameters are evaluated $A_{i X}$. If the lines of their level on the calibration portrait for $Y$ give all the points of intersection with the corresponding curves $A_{i Y},()$, and with the same abscissae $\mathrm{C}^{\star}$, then $\mathrm{H}_{0}$ is correct, and $\mathrm{C}^{\star}$ is the concentration of $\mathrm{Y}$ in the units of the portrait (see Figure 13).

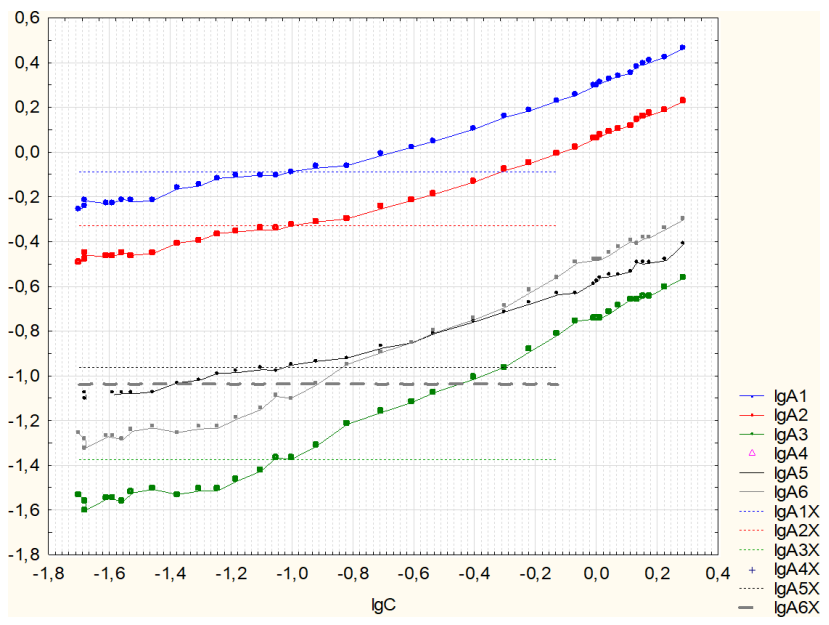

Figure 13. Detection of methane at a concentration of 0.109 vol.\% using a calibration portrait for a ZnO-based sensor.

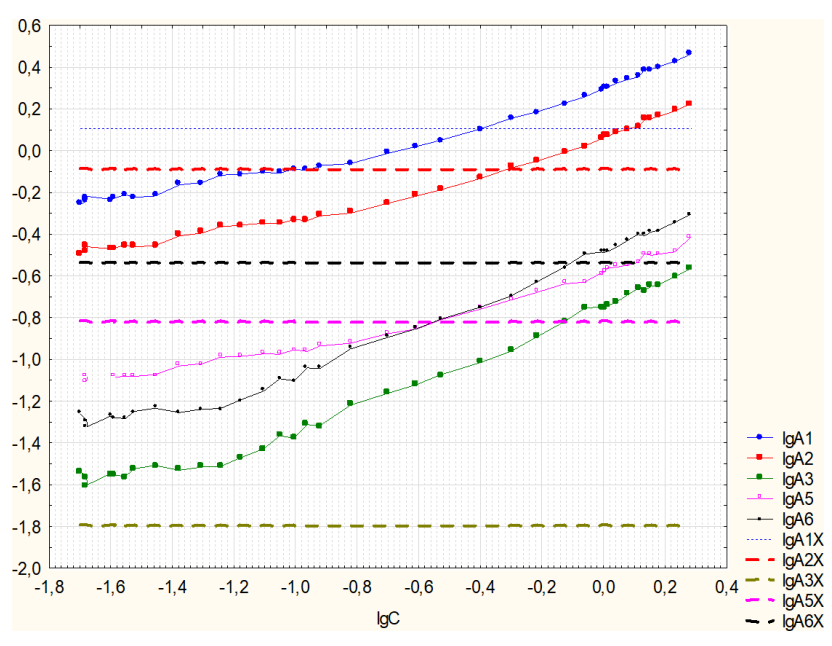

Figure 14. Complete discrepancy of 0.248 vol.\% propane in the air to the calibration portrait of methane on $\mathrm{ZnO}$-based sensor.

In the case of no/explicit difference of abscissa (see Figure 14), an alternative hypothesis $\mathrm{H}_{1}$ : $\mathrm{X} \neq \mathrm{Y}$ is accepted, then the following assumption for $Z$ is checked, etc.

Portraits are mainly used to recognize the chemical nature of the gas. For example, for nitric oxide $\mathrm{NO}$ in the air, all calibration lines start in «bald» sections (see Figure 15), which makes it difficult to calculate the concentration. In such cases the exact determination of the concentration can be carried out by an alternative method, for example, by the final electrical conductivity at a certain temperature (pre-calibration of this value by concentration is necessary) (Myasnikov et al. 1991).

If there are lines with a significant variation $A_{k Y},(C)$ in the current concentration range in the portrait, the inverse dependence $C\left(A_{k Y}\right)$, is estimated using nonlinear regres- 


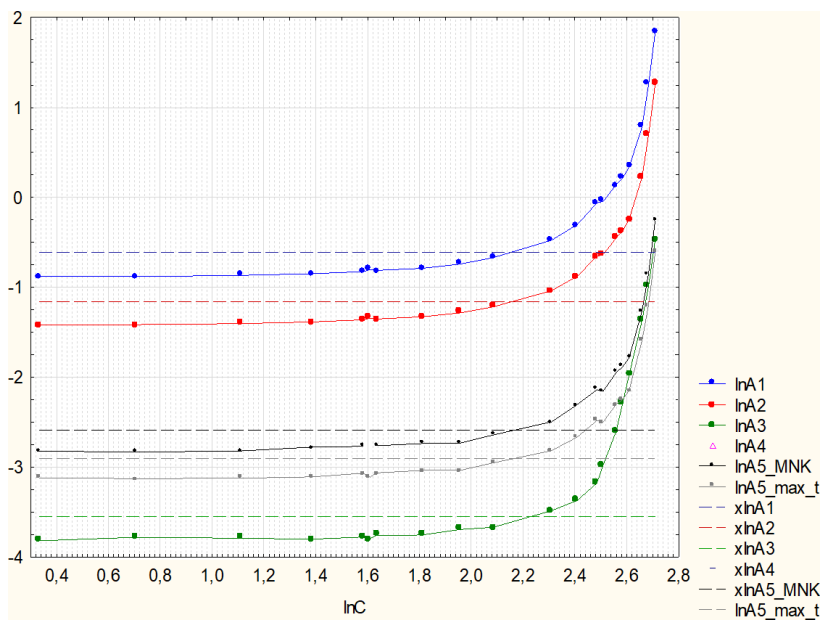

Figure 15. Recognition of $9.1 \mathrm{mg} / \mathrm{m}^{3} \mathrm{NO}$ in artificial air ( $\mathrm{ZnO}$-based sensor).

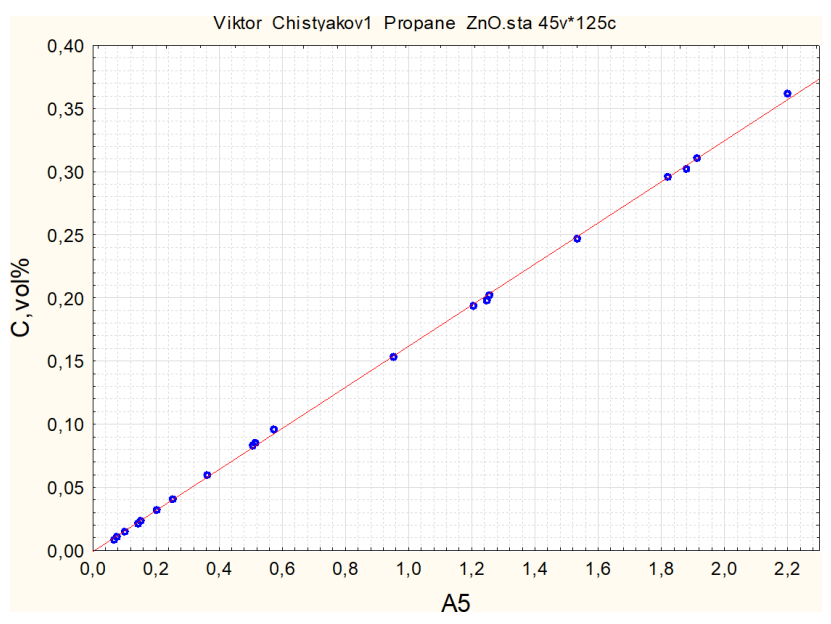

Figure 16. Ideal proportional dependence $C\left(A_{5}\right)$ for propane ( $\mathrm{ZnO}$ based sensor).

sion, which already serves as a calibration curve for finding the concentration.

As a rule, such inverse dependences have a power-law character with exponents from 1.5 to 1.7 , but for propane in the air, an ideal proportional dependence was observed within a wide range $C\left(A_{5}\right)$ (see Figure 16).

The developed method makes it possible to distinguish not only hydrocarbons of the same homology, but also various reducing particles: ammonia, nitric oxide, acetone, toluene, solvent and turpentine vapors. The method has great potential, since it is possible to vary the heating parameters, expand the range of interpolating functions, etc. In fact, the algorithm actually solves the most urgent problem of the selectivity of a conductometric sensor.

The use of single and rapid heating, as opposed to multiple cyclic heating, reduces both the measurement time and the wear of the sensor element. The check, which takes into account the drift of the sensor parameters due to a number of natural reasons, is carried out not on the identity of the estimated set of parameters $A_{i X}$ to a set $A_{i Y}$ of the same structure from the calibration portrait of the substance Y, but on the collinearity (proportionality) of these sets. In this case, the identification/exclusion criterion is a dimensionless value

$$
L_{\min }=\min _{C}\left(1-\frac{\left(\sum_{i} A_{i Y} A_{i X}\right)^{2}}{\left(\sum_{i} A_{i X}^{2}\right)\left(\sum_{i} A_{i Y}^{2}\right)}\right),
$$

exactly equal to zero with the exact proportionality of the sets $A_{i X}$ and $A_{i Y}$. Geometrically, this value is the square of the sine of the angle between the vectors $A_{i X}$ and $A_{i Y}$ in the multidimensional space of the main parameters. In the case where only $n=3$ parameters are significantly determined, $L_{\text {min }}$ is exactly the square of the sine of the angle between the velocity vectors of the molecules in an ideal gas.

A certain mathematical problem is the search for the type of distribution for $L_{\min }$ due to the requirement of positivity for $A_{i X}$ and $A_{i Y}$ and the lack of a priori assumptions about their distribution. Alternatively, the distribution table can be constructed experimentally, and the critical values of this parameter $L_{Y t h r}$ can be found at the standard level $95 \%$. Then if $L_{\min }<L_{Y t h r} \Rightarrow X=Y, C=\arg \left(L_{\min }\right)$. Otherwise $L_{\min } \geq L_{Y t h r} \Rightarrow X \neq Y$.

There is also an alternative way to check for collinearity of two vectors at a minimum $L$ (7) by estimating the proportionality coefficient $q$ of sets by simple regression $A_{i X}=$ $q A_{i Y}$. If the estimate produces a significant $q$ value and a high corrected coefficient of determination adj- $R^{2}>0.99$, then the sets are proportional. And then $\mathrm{X}$ is $\mathrm{Y}$.

\section{Discussion}

In the conditions of operation in space, the drift of the sensor parameters is largely due to the effect of radiation, so the choice of materials for gas-sensitive films is limited to those semiconductors whose structural features allow to neutralize the influence of high-energy particles. In REEdoped metal-oxide semiconductors and in some rare-earth semiconductors (for example, SmS), elements of variable valence can change their charge state under the influence of radiation. Apparently, in this process, the photoionization of two- or trivalent rare-earth ions occurs when excited within the bands of their f-d absorption. When the $5 \mathrm{~d}$ states of the donor are degenerate with the states of the conduction band, the probability of delocalization of an electron excited to such states becomes nonzero. The ability 
to give away an electron is not only possessed by donors in a stable valence form, which are realized in the crystal during synthesis. The activator ions are also photoionized in the metastable charge state obtained as a result of their radiation-stimulated reduction. This probably means that in such radiation-reduced donors, the excited $5 \mathrm{~d}$ states are located in the conduction band of the crystal (Brehovskih et al. 1982).

The algorithm of the measurement process is quite simple and convenient for practical use. This is a kind of "temperature scanner" of the gas-sensitive sensor layer in the measured atmosphere.

In the method described in Section 3.1 using the Fourier transform, the concentration "peak" observed on the graphs corresponds to the time when the change in the difference signal of the conductivity reaches the extremum point. The proportionality coefficient at the determined peak exit point in the system of presented calibration characteristics is the actual equilibrium constant. It is defined as $k_{1} / k_{2}$, where $k_{1}$ is the adsorption rate constant, and $k_{2}$ is the desorption rate constant of the impurity on the surface of the semiconductor. The peak exit point corresponds to the optimal detection temperature (Kazakov et al. 2019) determined with an error of about $1^{\circ} \mathrm{C}$. The desorption constant $k_{2}=\omega$ is found from the processing of the Fourier image of the signal, the constant $k_{1}$ is determined from the square of the real part of the spectral characteristic of the sensor $S(\omega)$. There is an unambiguous relationship between the relative electrical conductivity of the gas-sensitive element and the concentration of the impurity contained in the atmospheric air, which fully corresponds to the ideas about the mechanism of adsorption of active particles, discussed in detail in (Myasnikov et al. 1991). This circumstance makes it possible to determine the concentration of the measured component of the gas mixture according to the proposed system of calibration constants of the sensor.

The method described in Section 3.2 essentially solves the problem of the selectivity of the response of a chemical sensor even to substances of the same homologous group, such as methane, propane, and hexane. Data processing of electrophysical measurements by the nonlinear regression method is based on the Levenberg-Marquardt (LMA) algorithm. Nonlinear LMA estimation has never been considered a simple computational problem because of the difficulties of the initial assumption, the possible huge number of iterations or even cycles, as in the simplex method, multiple and close to each other solutions, etc. To overcome these difficulties, artificial intelligence (AI) and bioinspired methods have been used traditionally. The authors managed to avoid difficulties by creating initial assumptions databases for each sensitive element-substance pair. This was a complex numerical problem due to the obvious impossibility of a complete iteration with any acceptable grid. However, the type of functions (1)-(6) made it possible, fortunately, to solve this problem by simply converting to linear formulas by replacing $\Delta \sigma$ with $1 / \Delta \sigma$ and recalculating the obtained coefficients. This procedure, initially applied for large concentrations, gave the initial assumptions for LMA. The results obtained then served as the next initial guess for the lower concentration, etc.

\section{Conclusions}

The algorithms for processing data on the electrical conductivity of semiconductor sensors considered in the article successfully cope with the tasks of determining impurities in the atmospheric air, and the possibilities and resources of these approaches are far from exhausted both from the point of view of optimization and from the point of view of using artificial intelligence. At the same time, the proposed method for obtaining a signal (transient temperature modulation) with its representation in physical coordinates and the processing method are superior in selectivity to all currently known methods (Hosseini-Golgoo et al. 2017; Chen et al. 2017; Sakumura et al. 2017). Materials used as sensitive layers of sensors have characteristics characteristic of radiation-resistant materials: thermal stability, fine dispersion, and a large number of stoichiometric vacancies. High radiation resistance makes them promising for use as working layers of gas-sensitive sensors for express analysis of rocket fuel in space conditions.

Acknowledgment: This research was supported by Russian Foundation for Basic Research under grant 19-08-00576 A.

Author contribution: Sergey Kazakov: Writing - review \& editing, Data curation, Investigation, Methodology, Formal Analysis; Mikhail Grevtsev: Writing - original draft, Writing - review \& editing, Investigation; Victor Chistyakov: Writing - original draft, Data curation, Investigation, Formal Analysis; Sergey Solov'ev: Funding acquisition, Project administration, Writing - review \& editing, Resources.

Conflict of interest: Authors state no conflict of interest. 


\section{References}

Alizadeh T. 2010. Chemiresistor sensors array optimization by using the method of coupled statistical techniques and its application as an electronic nose for some organic vapors recognition. Sens Actuators B Chem. 143(2):740-749.

Brehovskih SM, Victorova YuN, Landa LM. Radiation effects in glasses. Moscow: Energoizdat; 1982. 182 p.

Chen J, Pan X, Boussaid F, McKinley A, Fan Z, Berm A. 2017. Breath Level Acetone Discrimination Through Temperature Modulation of a Hierarchical ZnO Gas. IEEE Sens Lett. 1(5):2000105.

Chistyakov VV, Kazakov SA, Grevtsev MA, Solovyev SM. 2021. Nonlinear regression algorithm for processing signals from semiconductor chemical sensors providing selective detection of impurities in artificial air. Tech Phys Lett. 47(3):275-279.

Gutman EY, Myasnikov IA, Kazakov SA, Rugentsev SV, Dymenko SK. 1993. The kinetic semiconductor gas-sensor conduction model and its practical use in gas analysis. Sens Actuators B Chem. 14(1-3):687-689.

Gwiźdź P, Brudniak A, Zakrewska K. Hydrogen Detection With a Gas Sensor Array - Processing and Recognition of Dynamic Responses Using Neural Networks. 2015. Metrol Meas Syst. 22(1):3-12.

He A, Wei G, Yu J, Tang Z, Lin Z, Wang P. 2017. A novel dictionary learning method for gas identification with a gas sensor array. IEEE Trans Ind Electron. 64(12):9709-9715.

Hosseini-Golgoo SM, Salimi F, Saberkari A, Rahbarpour S. 2017. Comparison of information content of temporal response of chemoresistive gas sensor under three different temperature modulation regimes for gas detection of different feature reduction methods. J Phys Conf Ser. 939:012005.
Kaminsky VV, Vasilyev LN, Gornushkina ED, Solovyev SM, Sosova GA, Volodin NM. 1995. The influence of gamma irradiation on electrical parameters of thin SmS films. Semiconductors. 29(2):306-308.

Kazakov SA, Kaminsky VV, Solovyev SM, Sharenkova NV. 2015. Semiconductor gas oxygen sensors based on polycrystalline films of samarium sulfide. Nauchnoe Priborostroenie. 25(3):116-123.

Kazakov SA, Grevtsev MA, Sokolov AV, Kaminsky VV, Kazanin MM. 2019. Semiconductor gas sensors of gasoline and solvent vapors concentrations based on SmS polycrystalline films. Nauchnoe Priborostroenie. 29(4):80-83

Kazakov SA, Grevtsev MA, Khavrov GD. Calibration algorithm for semiconductor gas sensors and determination of volatile hydrocarbon concentrations in atmospheric air using Fourier transforms. J Phys Conf Ser, 1697 012243, International Conference PhysicA.SPb/2020. 2020 19-23 Oct; Saint Petersburg, Russia. https://doi.org/10.1088/1742-6596/1697/1/012243.

Myasnikov IA, Sukharev VYa, Kupriyanov LYu, Zav'yalov SA. Semiconductor Sensors in Physicochemical Investigations. Moscow: Nauka; 1991. 327 p.

Pijolata C, Tourniera G, Breuila P, Matarinb D, Nivetb P. 2002. Hydrogen detection on a cryogenic motor with a $\mathrm{SnO}_{2}$ sensors network. Sens Actuators B Chem. 82(2-3):166-175.

Sakumura Y, Koyama Y, Tokutake H, Hida T, Sato K, Itoh T, et al. 2017. Diagnosis by volatile organic compounds in exhaled breath from lung cancer patients using support vector machine algorithm. Sensors (Basel). 17(2):287.

Shahid A, Choi J-H, Rana AUHS, Kim H-S. 2018. Least squares neural network-based wireless E-Nose system using an $\mathrm{SnO}_{2}$ sensor 60 array. Sensors (Basel). 18(5):1446. 